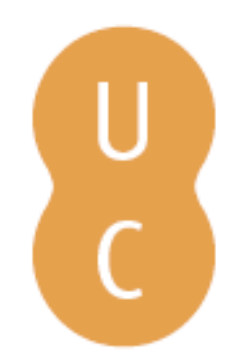

\title{
nombalina
}

\section{Sistema de observação da aliança em terapia familiar versão observacional (SOFTAO)}

\author{
Autor(es): $\quad$ Sotero, Luciana; Relvas, Ana Paula \\ Publicado por: Imprensa da Universidade de Coimbra \\ URL \\ persistente: URI:http://hdl.handle.net/10316.2/35902 \\ DOI: $\quad$ DOI:http://dx.doi.org/10.14195/978-989-26-0839-6_5 \\ Accessed : $\quad$ 26-Apr-2023 03:49:47
}

A navegação consulta e descarregamento dos títulos inseridos nas Bibliotecas Digitais UC Digitalis, UC Pombalina e UC Impactum, pressupõem a aceitação plena e sem reservas dos Termos e Condições de Uso destas Bibliotecas Digitais, disponíveis em https://digitalis.uc.pt/pt-pt/termos.

Conforme exposto nos referidos Termos e Condições de Uso, o descarregamento de títulos de acesso restrito requer uma licença válida de autorização devendo o utilizador aceder ao(s) documento(s) a partir de um endereço de IP da instituição detentora da supramencionada licença.

Ao utilizador é apenas permitido o descarregamento para uso pessoal, pelo que o emprego do(s) título(s) descarregado(s) para outro fim, designadamente comercial, carece de autorização do respetivo autor ou editor da obra.

Na medida em que todas as obras da UC Digitalis se encontram protegidas pelo Código do Direito de Autor e Direitos Conexos e demais legislação aplicável, toda a cópia, parcial ou total, deste documento, nos casos em que é legalmente admitida, deverá conter ou fazer-se acompanhar por este aviso.

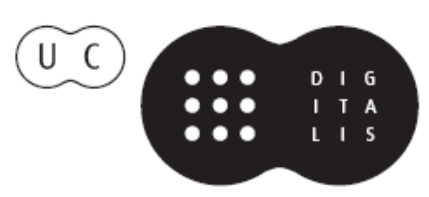




\title{
AVALIAÇÃO FAMILIAR IN IVREVENGGio \\ FUNCIONAMENTO E VOL. I FAMILIAR
}

(2) 1.57

\begin{abstract}
Vol. 1
\end{abstract}

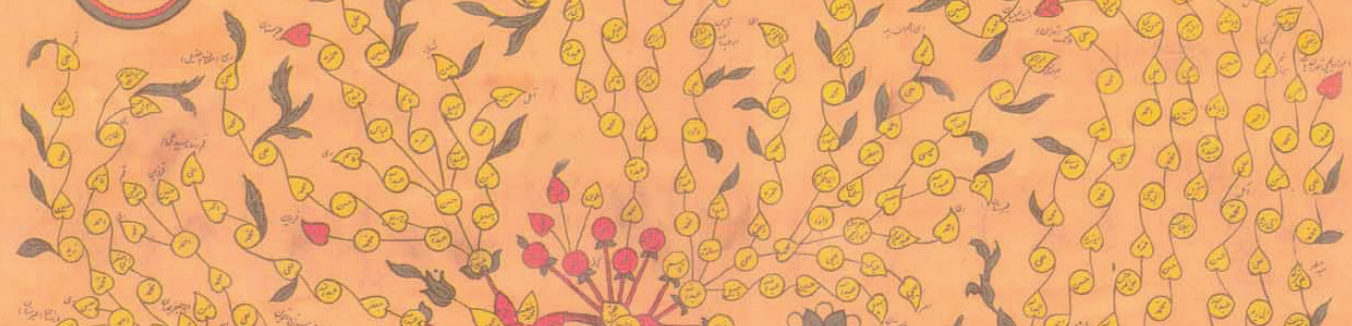
a

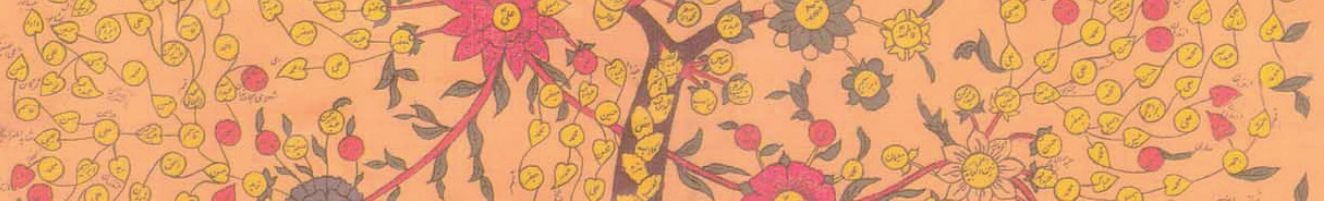

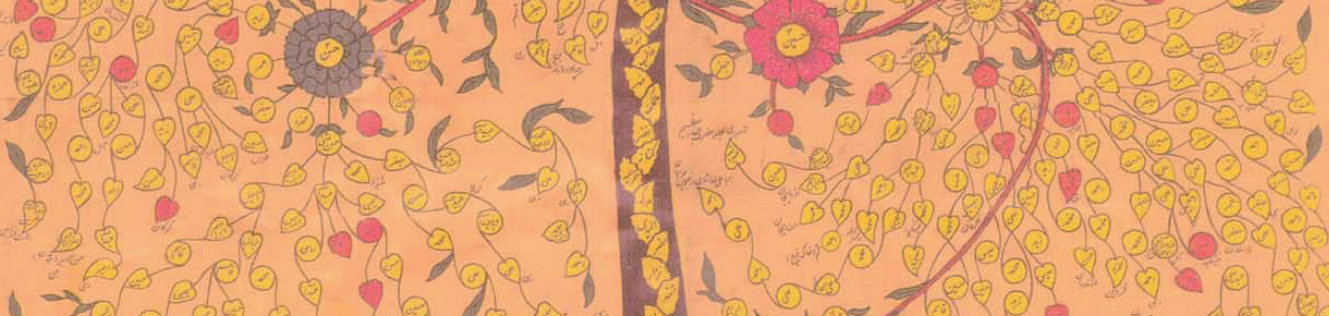

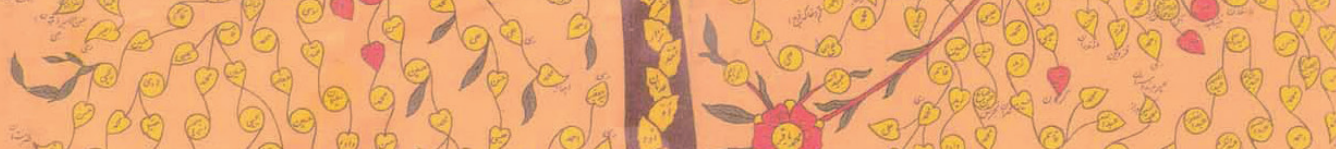
792.00

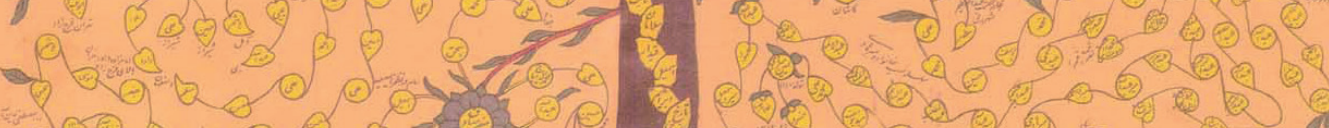

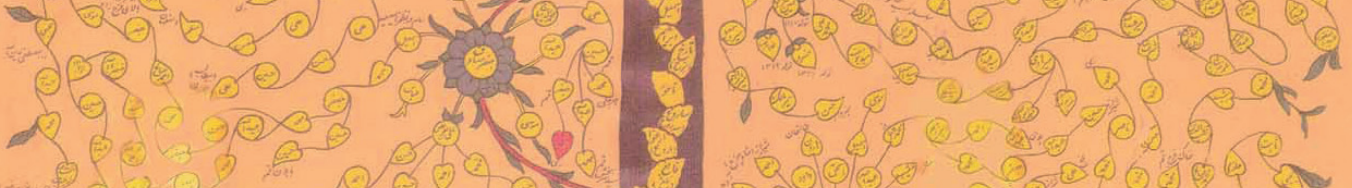

$1070-605$

ANA PAULA RELVAS UU, H(COOŔDENACÃO) 


\section{SISTEMA DE OBSERVAÇÃO DA ALIANÇA EM TERAPIA F A M I L I A R \\ VERSÃOO BSERVACIONAL \\ ( S O F TA - O)}

Luciana Sotero Ana Paula Relvas

"...because we believe that a good alliance can be seen..."

(Friedlander, Escudero, \& Heatherington, 2006, p. 263)

\section{Resumo}

A versão Portuguesa do System for Observing Family Therapy Alliances (SOFTA; Friedlander, Escudero, \& Heatherington, 2006), versão observacional (SOFTA-o), foi desenvolvida com o objetivo de fomentar a investigação e uma prática clínica melhor informada acerca da aliança terapêutica na terapia familiar. A partir deste sistema de codificação de comportamentos observáveis durante as sessões de terapia é possível avaliar a força da aliança terapêutica em duas dimensões comuns às diferentes modalidades terapêuticas (Envolvimento no Processo Terapêutico e Conexão Emocional com o Terapeuta) e em duas dimensões específicas das terapias conjuntas (Segurança Dentro do Sistema Terapêutico e Sentimento de Partilha de Objetivos na Família). As propriedades psicométricas do instrumento são evidenciadas em três estudos de fiabilidade (acordo interobservadores) e três estudos de validade (convergente, preditiva e grupos-conhecidos). 
Os resultados alcançados são encorajadores da utilização da versão portuguesa do SOFTA-o na investigação, prática clínica e formação, treino e supervisão de terapeutas.

Palavras-chave: aliança terapêutica, SOFTA-o, comportamentos observáveis, estudos psicométricos.

\section{Abstract}

The Portuguese version of the System for Observing Family Therapy Alliances (SOFTA; Friedlander, Escudero, \& Heatherington, 2006), observational version (SOFTA-o), was developed in order to promote research and a clinical practice better informed about the therapeutic alliance in family therapy. With this coding system of observable behaviors during therapy sessions it is possible to assess the strength of the therapeutic alliance in two dimensions that are common across therapy modalities (Engagement in the Therapeutic Process and Emotional Connection to the Therapist) and two specific dimensions of conjoint therapies (Safety Within the Therapeutic Context and Shared Sense of Purpose within the Family). The psychometric support of the instrument is provided by three reliability studies (interobserver agreement) and three validity studies (convergent, predictive and known-groups). The results achieved encourage the use of the Portuguese version of SOFTA-o in research, clinical practice, training and supervision of therapists.

Keywords: therapeutic alliance, SOFTA-o, observational behaviors, psychometric studies. 


\title{
1. Instrumento
}

\section{O que é, o que avalia e a quem se aplica?}

\author{
No Quadro 1 encontra-se a ficha técnica relativa ao Sistema de \\ Observação da Aliança em Terapia Familiar, versão observacional (SOFTA- \\ -o; Friedlander, Escudero, \& Heatherington, 2006).
}

Quadro 1.

Ficha técnica do SOFTA-o

\begin{tabular}{ll} 
& O Sistema de Observação da Aliança em Terapia Familiar é a versão \\
& portuguesa, para clientes e terapeutas, do System for Observing Family \\
& Therapy Alliances (SOFTA) publicado em 2006 por Friedlander, Es- \\
O que é? & cudero e Heatherington. O SOFTA é constituído por um conjunto de \\
& ferramentas: SOFTA-o (versão observacional), SOFTA-s (versão auto- \\
& -resposta) e um programa informático (e-SOFTA) que facilita o treino \\
& de investigadores e clínicos na utilização do instrumento (Disponível \\
& em http://www.softa-soatif.com) \\
\hline O SOFTA versão observacional (SOFTA-o) foi desenvolvido com o \\
objetivo de avaliar a força da aliança terapêutica a partir de comporta- \\
mentos observáveis durante as sessões de terapia familiar ou de casal. É \\
constituído por 44 itens, ou descritores comportamentais, positivos e ne- \\
gativos, verbais e não verbais, que permitem estimar a força da aliança \\
em quatro dimensões específicas: Envolvimento no Processo Terapêti- \\
co, Conexão Emocional com o Terapeuta, Segurança Dentro do Sistema \\
Terapêutico e Sentimento de Partilha de Objetivos na Família
\end{tabular}

Estrutura do SOFTA-o

\begin{tabular}{lll}
\hline Subescala & Número Itens & Descrição \\
\hline & & Reflete a coopera- \\
& ção e o envolvi- \\
& mento do cliente \\
Envolvimento no Processo & 11 & na terapia (e.g., "O \\
Terapêutico (ENV) & & cliente manifesta o \\
& seu acordo com as \\
& metas propostas \\
& pelo terapeuta")
\end{tabular}

Retrata a forma como o cliente vê o terapeuta e a

Conexão Emocional com o Terapeuta

(CE)
10 relação que tem com este (e.g., "O cliente partilha um momento de humor ou uma brincadeira com o terapeuta" 
Segurança dentro do Sistema

Terapêutico (SEG)
12
11

Representa a segurança que o cliente sente (ou não) para assumir riscos e falar abertamente na terapia (e.g., "O cliente revela um segredo ou algo que nenhum membro da família sabe")

Avalia o sentimento de unidade e de colaboração no seio da família face ao

Sentimento de Partilha de Objetivos na Família (SPO) valor e propósito

da terapia (e.g.,

"Os membros da

família perguntam uns aos outros os pontos de vista de cada um")

O SOFTA-o foi desenvolvido para ser utilizado durante a observação direta, ou a gravação em vídeo, de uma sessão de terapia familiar ou de casal (com um mínimo de dois participantes)

O acesso ao SOFTA-o (versão clientes e versão terapeutas) e ao Manual de Treino pode ser efetuado através da página http://www.fpce. uc.pt/avaliaçaofamiliar que contém todos os instrumentos de avaliação

Como ter acesso? apresentados neste livro. Os utilizadores deverão facultar os contactos pessoais e institucionais, bem como dados acerca do propósito da utilização do SOFTA-o (e.g., investigação, prática clínica) e concordar com as condições de utilização e de partilha dos resultados com os autores da versão portuguesa

\section{Fundamentação e bistória}

Décadas de investigação empírica acerca da aliança terapêutica permitem afirmar que esta é uma característica crucial da terapia (e.g., Goldfried, 1980; Luborsky, Singer, \& Luborsky, 1975; Rogers \& Wood, 1974). Segundo Horvath, Del Re, Flückiger e Symonds (2011), o interesse sustentado da comunidade científica na aliança terapêutica ficou provavelmente a dever-se à descoberta consistente de uma relação robusta entre a aliança e os resultados da terapia, em diferentes modalidades de intervenção e numa diversidade de clientes e problemas (e.g., Horvath \& Bedi, 2002; Horvath \& Symonds, 1991; Martin, Garske, \& Davis, 2000). 
Contudo, a maioria da investigação tem sido desenvolvida no contexto da terapia individual e pouco se sabe ainda acerca da construção e manutenção de uma aliança forte nas terapias conjuntas (i.e., na terapia familiar e de casal) (Friedlander et al., 2006). De facto, a falta de conhecimentos sobre a aliança em terapia familiar não é por acaso e está associada a uma dificuldade adicional nesta modalidade de terapia: compreendê-la e medi-la num sistema complexo multipessoal e multigeracional. Assim, para avaliar a aliança em terapia familiar e de casal é preciso atender a dimensões que são comuns às diferentes modalidades terapêuticas e que correspondem à conceptualização clássica de Bordin (1979); ligação afetiva entre cliente e terapeuta e acordo entre ambos sobre os objetivos e tarefas da terapia, mas também requer atenção a dimensões que refletem a singularidade das terapias conjuntas. Apesar das componentes da aliança anteriormente referidas (Bordin, 1979) fazerem sentido na terapia individual, no contexto particular da terapia familiar as relações desenvolvidas não são limitadas ao binómio cliente-terapeuta.

Pinsof (1994, 1995; Pinsof \& Catherall, 1986) foi pioneiro na investigação e conceptualização sistémica da aliança terapêutica, tendo sido o primeiro autor a descrever a causalidade recíproca da aliança na intervenção familiar, argumentando que esta é mais do que o resultado da soma das alianças individuais (The whole is more than the sum of its parts; Bertalanffy, 1972, p. 417). Na terapia familiar e de casal todos os clientes observam a interação dos restantes com o terapeuta, o que gera um sistema complexo de influências recíprocas, enquanto na abordagem terapêutica individual a relação entre cliente e terapeuta é apenas bidirecional. Isto significa que a aliança do terapeuta com cada um dos elementos afeta e é afetada pela aliança que estabelece com todos os outros elementos da família. Durante a sessão, cada cliente observa de perto como os outros membros da família falam e interagem com o terapeuta, o que pode determinar o sucesso ou o fracasso do trabalho conjunto (Não apenas a parte está no todo como o todo está na parte; Morin, 1991, p. 90).

Outro aspeto relevante no estudo da aliança nas terapias conjuntas, diz respeito à aliança estabelecida no seio da própria família, descrita na literatura inicialmente como "aliança dentro-da-família" (within-family 
aliance, Pinsof, 1994), posteriormente como "lealdade" (allegiance, Symonds \& Horvath, 2004) e mais recentemente como "sentimento de partilha de objetivos dentro da família" (shared sense of purpose within the family, Friedlander et al., 2006). Esta dimensão não se refere apenas ao desejo de colaborar no tratamento, mas também a uma ligação forte entre os membros da família. Na terapia individual a força da aliança depende exclusivamente das contribuições mútuas do cliente e terapeuta. Porém, no contexto das terapias familiares deve ser considerada a partir de uma visão sistémica, isto é, depende não apenas da relação entre os clientes e o terapeuta mas também das inter-relações entre os próprios clientes (Falloon, 1991). Neste sentido, vários estudos têm demonstrado que a família ou o casal são a unidade mais influente em termos de motivação para a mudança, colocando a mudança em marcha (Pinsof, 1994) e influenciando os resultados terapêuticos finais (Knobloch-Fedders, Pinsof, \& Mann, 2004; Robbins, Turner, Alexander, \& Perez, 2003; Symonds \& Horvath, 2004).

A singularidade da aliança nas terapias conjuntas é ainda legitimada pela existência de um fenómeno único desse tipo de terapias designado por "alianças cindidas" (slipt alliances, Heatherington \& Friedlander, 1990; Pinsof \& Catherall, 1986) ou "alianças desequilibradas" (unbalanced aliances, Robbins et al., 2003), o que acontece quando os membros da família diferem significativamente nas suas atitudes face à terapia ou ao terapeuta. As alianças divididas ou cindidas são bastante frequentes na terapia com a família (Pinsof \& Catherall, 1986; Robbins et al., 2003) e podem interferir profundamente no processo terapêutico (Heatherington \& Friedlander, 1990; Muñiz de la Peña, Friedlander, \& Escudero, 2009). A investigação mostra que as discrepâncias nas alianças dos clientes com o terapeuta podem interferir na terapia mais do que qualquer aliança individual considerada separadamente (Robbins et al., 2006; Robbins et al., 2003; Symonds \& Horvath, 2004). Um caso particularmente difícil neste permanente balanceamento das alianças, diz respeito à terapia com famílias com filhos adolescentes, sendo frequente encontrar relações terapêuticas mais positivas com os pais do que com os filhos (Robbins et al., 2003), mas o contrário também é possível (Muñiz de la Peña et al., 2009), contribuindo, ambos os casos, para um risco acrescido de abandono da terapia (dropout). 
É igualmente importante reconhecer que, para os clientes, os riscos de fazer uma terapia familiar são consideravelmente superiores do que fazer uma terapia individual, uma vez que a informação revelada nesta última modalidade pode não trazer consequências diretas na vida familiar (Friedlander et al., 2006). Em contrapartida, nas terapias conjuntas a partilha de informações novas no espaço terapêutico (e.g., segredos familiares, não ditos) pode resultar em consequências negativas para algum ou alguns dos membros da família e/ou na deterioração das relações, entre outros efeitos indesejáveis. Na terapia individual, é escolha do cliente o que revelar e quando, enquanto nas terapias conjuntas os clientes têm pouco ou nenhum controlo sobre o que os outros escolhem revelar (Escudero, Heatherington, \& Friedlander, 2010; Friedlander et al., 2006). Assim, o que acontece nas sessões de terapia familiar pode ter consequências efetivas no sistema familiar, principalmente se o terapeuta não for capaz de gerir os conflitos que surgem durante a sessão. O grau de segurança sentido pelos elementos da família pode ainda mudar à medida que novos problemas são explorados e quando diferentes elementos se juntam ou deixam a terapia (Beck, Friedlander, \& Escudero, 2006), flutuações estas às quais o terapeuta deve também estar atento.

Segundo Rait (2000), o maior desafio dos terapeutas familiares é construir e manter alianças terapêuticas a múltiplos níveis simultaneamente, isto é, com cada um dos elementos familiares, com os vários subsistemas (e.g., conjugal ou fraternal) e com a família como um todo. Estabelecer e gerir múltiplas alianças em simultâneo pode ser particularmente complexo por motivos diversos: a presença de diferentes níveis desenvolvimentais dos elementos familiares (e.g., crianças, adolescentes, adultos), os segredos e agendas ocultas (e.g., traições, desejo de separação conjugal), as histórias de conflitos e mal-entendidos, diferentes objetivos ou concepções distintas de como mudar e diferentes níveis motivacionais, sendo também frequente encontrar quem não queira estar na terapia (Friedlander et al., 2006; Rait, 2000; Sotero \& Relvas, 2012).

$\mathrm{Na}$ terapia familiar e de casal, estas observações revelam que entre clientes e terapeutas é essencial uma ligação afetiva forte e um acordo mútuo acerca dos objetivos e tarefas da terapia, tal como acontece na 
terapia individual, mas os terapeutas familiares têm igualmente de prestar atenção a aspetos únicos e interrelacionados com esta modalidade terapêutica (Friedlander et al., 2006), nomeadamente o grau em que os membros da família concordam entre si sobre a necessidade, finalidade e valor da terapia (Pinsof, 1994; Rait, 2000); e o grau em que as pessoas se sentem seguras a discutir os problemas com outros membros da família (Christensen, Russell, Miller, \& Peterson, 1998).

Neste sentido, a questão da conceptualização da aliança nas terapias conjuntas é também uma questão empírica, fazendo sentido questionar como se pode combinar a avaliação da aliança dos diferentes elementos familiares; como medir a aliança terapêutica dentro da família; ou se a aliança de alguns elementos familiares será mais importante do que a de outros.

Antes do System for Observing Family Therapy Alliances (SOFTA; Friedlander et al., 2006), os primeiros e únicos questionários de auto-resposta especificamente desenvolvidos para avaliar a aliança nas terapias conjuntas são da autoria de Pinsof e Catherall (1986): A Couple Therapy Alliance Scale (CTAS) e a Family Therapy Alliance Scale (FTAS), também designadas conjuntamente por Integrative Psychotherapy Alliance Scales. Estes questionários são baseados na operacionalização tripartida da aliança (Bordin, 1979) - três conteúdos distintos (objetivos, tarefas, ligação afetiva) - e em três dimensões interpessoais (terapeuta-cliente; terapeuta-família; terapeuta-subsistemas). Mais tarde, Pinsof (1994, 1995) acabou por acrescentar uma quarta dimensão interpessoal - a aliança dentro do próprio sistema: quer familiar (aliança entre os elementos familiares), quer terapêutico (aliança entre co-terapeutas, e/ou supervisores, equipa de observação, instituições). Recentemente, Pinsof, Zinbarg e Knobloch-Fedders (2008) desenvolveram uma versão reduzida das escalas (CTASr e FTASr), com vista a facilitar a sua utilização na prática clínica e investigação.

Um outro instrumento bastante utilizado na avaliação da aliança em terapia de casal é o Working Alliance Inventory - Couples (WAI-C; Symonds, 1998 citado em Friedlander et al., 2006; Symonds \& Horvath, 2004). Contudo, este instrumento não foi especificamente criado para as terapias conjuntas, tendo sido adaptado a partir da sua versão original para a terapia individual (WAI; Horvath \& Greenberg, 1986). 
Para além da escassez de instrumentos nesta área, as escalas disponíveis são todas de auto-resposta, o que possibilita avaliar a aliança questionando clientes e/ou terapeutas, mas apenas permite aceder à perspetiva interna dos informadores sobre o que se passa na terapia. A maioria da investigação existente baseia-se assim em questionários que medem o processo intrapessoal da aliança, a partir de um ponto de vista individual, e pouco se sabe sobre quais os comportamentos de clientes e terapeutas que refletem a experiência da aliança durante a sessão. Em alternativa, as medidas observacionais surgem como particularmente úteis, neste contexto, ao refletirem o processo clínico e os fenómenos interpessoais (Escudero et al., 2010), permitindo avaliar, momento a momento, por parte de um observador externo, a aliança terapêutica através de comportamentos observáveis, dos clientes e terapeutas, à medida que o processo terapêutico decorre. Possibilitam ainda monitorizar, em tempo real, as flutuações da aliança e relacioná-las com as intervenções terapêuticas (e.g., reenquadramentos, conotações positivas, técnicas ativas) e os acontecimentos (e. g., discussões, revelações, ruturas) durante a sessão.

$\mathrm{Na}$ literatura atual há referência a dois sistemas observacionais de avaliação da aliança: 1) a Vanderbilt Therapeutic Alliance Scale (VTAS-R) adaptada ao contexto das terapias conjuntas por Diamond, Liddle, Hogue, e Dakof (1999), a partir da versão original criada para a psicoterapia individual; e 2) o System for Observing Family Therapy Alliances (SOFTA-o; Friedlander et al., 2006), o primeiro instrumento especificamente desenvolvido para observar os comportamentos que refletem a aliança, de clientes e terapeutas, no contexto particular das terapias conjuntas. O SOFTA-o surgiu da vontade de criar um modelo multidimensional da aliança que refletisse o que esta tem de comum com outras modalidades terapêuticas e de específico na terapia familiar e de casal.

O SOFTA-o versão clientes foi o primeiro a ser desenvolvido, com o objetivo de obter uma ferramenta que pudesse informar sobre os comportamentos dos clientes que contribuem para a força da aliança terapêutica. Inicialmente, para a construção do instrumento, os autores realizaram uma revisão teórica, clínica e empírica da literatura sobre a relação terapêutica em terapia familiar e de casal. Este processo, complementado 
pela extensa experiência clínica dos autores, resultou na identificação de um conjunto de indicadores comportamentais que ilustram tanto uma aliança positiva (e.g., "O cliente indica que se sente entendido ou aceite pelo terapeuta"), como negativa (e.g., "O cliente evita o contacto ocular com o terapeuta"). Estes descritores comportamentais foram então utilizados para criar uma primeira lista que ilustrasse diferentes aspetos e níveis diferenciados de participação e colaboração dos clientes nas terapias conjuntas. Na construção dos descritores foi tomado como critério essencial que se tratassem de comportamentos observáveis. Por exemplo, em vez da operacionalização "os membros da família estão interessados nos pontos de vista uns dos outros" utiliza-se o descritor "os membros da família perguntam uns aos outros sobre os pontos de vista de cada um".

Para afinar o inventário de descritores, uma equipa de quatro investigadores analisou 12 sessões de terapia familiar gravadas em vídeo, nas quais os clientes tinham preenchido as Integrative Psychotherapy Alliance Scales (Pinsof \& Catherall, 1986). Conhecendo, portanto, a perspetiva de cada membro da família sobre a aliança, analisaram-se as gravações das sessões para identificar comportamentos interpessoais que pudessem ser claramente indicativos dos seus sentimentos e pensamentos acerca do que ocorria durante a sessão. Assim, foram-se refinando e editando os descritores, através de comparações sucessivas de cada uma das observações feitas. As discrepâncias serviram para clarificar os descritores e excluir aqueles que seriam especialmente difíceis de codificar ou pontuar.

Para criar as quatro dimensões do SOFTA, a equipa de investigadores começou por agrupar os descritores similares e nomear os clusters resultantes. Posteriormente, cada investigador, de forma independente, desenvolveu as definições dos constructos refletidos em cada cluster. Comparando e integrando as definições, os investigadores decidiram depois quais os descritores que, de forma lógica, se relacionavam com cada uma das dimensões. Um estudo piloto a este procedimento de codificação, com seis vídeos diferentes, mostrou que o processo era viável e resultou num bom nível de concordância interobservadores.

$O$ passo seguinte foi estudar a validade facial (face validity) do instrumento através de uma tarefa de classificação (sorting task). Os 44 
descritores resultantes do processo de análise prévio foram ordenados aleatoriamente, pedindo-se a um grupo de investigadores $(N=24)$, dos Estados Unidos da América (EUA), Canadá e Espanha, peritos na análise do processo terapêutico em terapia familiar, para indicarem os descritores que refletiam melhor cada um dos quatro constructos subjacentes. Se pelo menos $75 \%$ dos especialistas que responderam à tarefa de classificação, selecionaram a mesma dimensão para um determinado descritor, esse descritor foi mantido entre os indicadores dessa mesma dimensão. Caso contrário, o descritor foi removido ou incluído numa dimensão diferente. Também foi pedido aos peritos que comentassem os descritores, com vista a incrementar a validade de conteúdo do SOFTA, designadamente, acrescentando outros descritores representativos de uma determinada dimensão. Os peritos espanhóis ( $N=7$, investigadores e terapeutas familiares prestigiados) foram ainda convidados a comentar a adequação cultural dos descritores e das dimensões às características culturais dos clientes e terapeutas no contexto da terapia familiar espanhola. Os resultados desta tarefa de classificação indicaram um elevado grau de consistência e a maioria dos descritores comportamentais foi classificada como representante da dimensão que fora previamente selecionada. A maioria dos descritores foi classificada da mesma forma, por mais de $75 \%$ dos juízes em ambas as amostras, revelando uma boa consistência (versão Norte-Americana $k s=.81$ e versão Espanhola $k s=.71$; Friedlander, Escudero, Horvath et al., 2006). Um total de apenas cinco descritores foi eliminado por preencher os critérios relativos à obtenção de um consenso de $75 \%$ entre os juízes numa única amostra (apenas para os EUA ou apenas para Espanha). Nenhum dos descritores teve que ser eliminado por ser pouco apropriado em termos de diferenças culturais e apenas foram introduzidas algumas mudanças mínimas na definição ou tradução de alguns deles.

A versão do SOFTA-o para o terapeuta foi desenvolvida através de um processo semelhante (Friedlander et al., 2005). Começou-se por identificar um conjunto de descritores, alguns dos quais paralelos aos que tinham sido identificados para os clientes. Em seguida, realizou-se um estudo de validade de conteúdo através de uma prova de classificação dos descritores com especialistas espanhóis e norte-americanos. Com 
base nos resultados dessa classificação foram adicionados, modificados ou excluídos alguns descritores.

Para testar as qualidades psicométricas do SOFTA-o foram desenvolvidos a nível internacional vários estudos, nomeadamente cinco estudos de fiabilidade, uma análise fatorial exploratória e estudos correlacionais com vários índices do processo e dos resultados terapêuticos (Friedlander, Escudero, Horvath et al., 2006). O sistema de observação apresentou valores adequados de fiabilidade (correlações intra-classe de .72 a .95) e a validade de constructo foi demonstrada através de análise fatorial, validade grupos-conhecidos (known-groups validity) e validade preditiva (Friedlander, Escudero, Horvath et al., 2006). Apesar de a análise fatorial exploratória ter identificado apenas um fator (i.e. aliança terapêutica), os autores do instrumento optaram por manter a conceptualização da aliança atendendo às quatro dimensões já referidas, uma vez que as intercorrelações entre as dimensões apresentaram uma amplitude considerável (de .18 a .75), parecendo indicar que as quatro dimensões do SOFTA não são excludentes, apesar de refletirem um constructo unitário (Friedlander, Escudero, Horvath et al., 2006). Os diversos estudos de validade demonstraram que (1) os piores resultados terapêuticos estão associados a uma menor Segurança e um menor Sentimento de Partilha nas sessões iniciais (Beck et al., 2006); (2) pontuações altas no SOFTA-o estão associadas a pontuações favoráveis da aliança no Self-Report Helping Alliance Questionnaire de Luborsky, Crits-Cristoph, Alexander, Margolis, e Cohen (1983) (Friedlander et al., 2006), uma maior profundidade da sessão (Friedlander, Bernardi, \& Lee, 2010) e melhorias no cliente (e.g., Escudero, Friedlander, Varela, \& Abascal, 2008); e (3) mesmo alianças cindidas ligeiras (split aliances) podem ser observadas através do SOFTA-o (Muñiz de la Peña et al., 2009). Todavia, os autores do instrumento recomendam que sejam realizados estudos adicionais que possam confirmar estes resultados (Friedlander et al., 2006).

Segundo o modelo conceptual do SOFTA (cf. Figura 1), as quatro dimensões são úteis para a prática clínica e refletem a definição operacional da aliança: a Segurança dentro do Sistema Terapêutico e o Sentimento de Partilha de Objetivos na Família espelham as especificidades da terapia com múltiplos clientes; enquanto o Envolvimento no Processo Terapêutico e a Conexão 
Emocional com o Terapeuta retratam as características comuns a todas as modalidades de tratamento (Bordin, 1979). De notar que estas dimensões não são mutuamente exclusivas. Assim, o sentido de segurança de um cliente está intimamente relacionado com a sua ligação emocional ao terapeuta e com o seu envolvimento na terapia. Tal como estas dimensões tendem a ser mais fortes quando toda a família está de acordo quanto às razões que os levam a estar na terapia e com o que esperam conseguir com a intervenção. Contudo, cada dimensão oferece informação específica e não redundante acerca da força da aliança terapêutica para um dado cliente ou família.

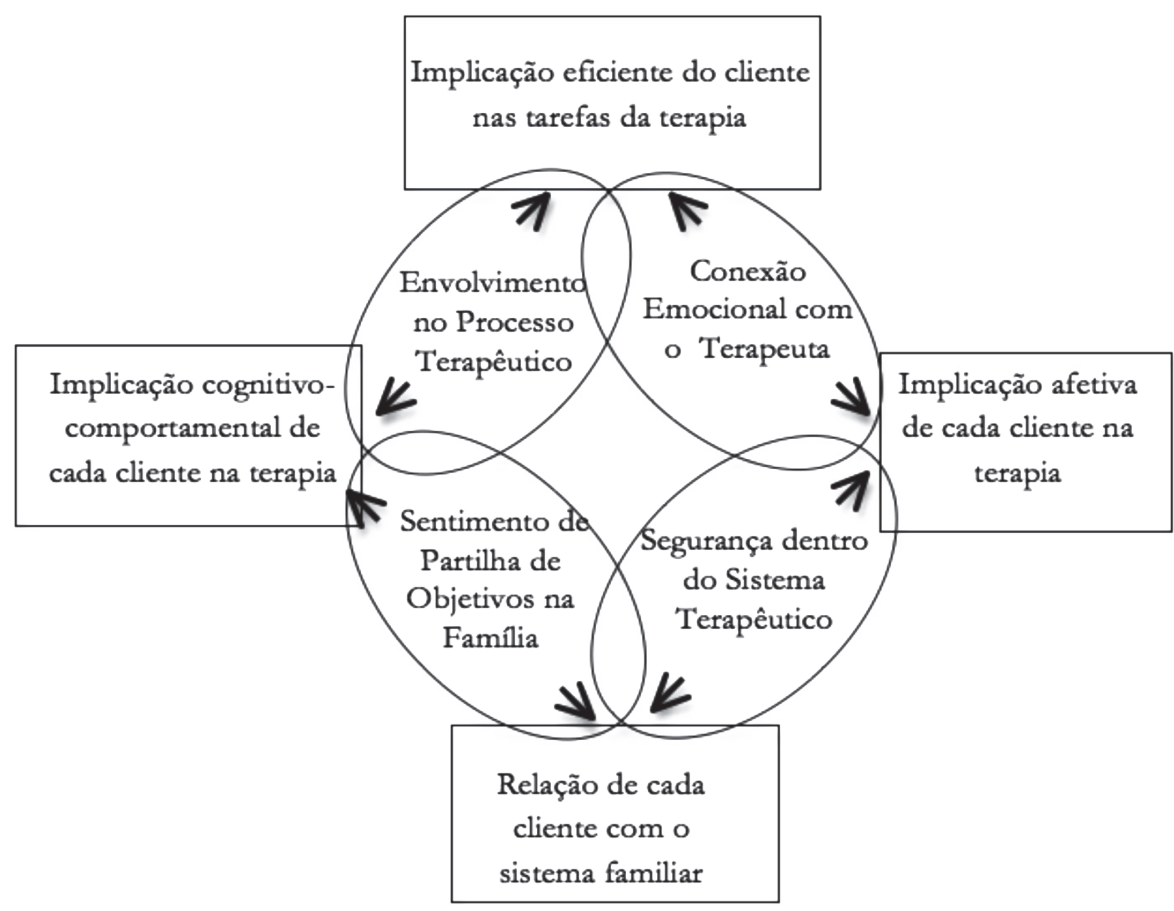

Figura 1. Inter-relações das dimensões do SOFTA. Adaptado de "La creación de la alianza terapêutica en la terapia familiar", de V. Escudero, 2009, Apuntes de Psicologia, 27, p. 255. Adaptado com permissão.

Resumindo, o SOFTA-o permite avaliar a força da aliança terapêutica a partir de comportamentos observáveis, baseando-se num modelo multidimensional e transteórico do constructo: a) multidimensional porque para além das quatro dimensões que definem operacionalmente a alian- 
ça, estão representados quer o sistema cliente, quer o sistema terapeuta, com dimensões que refletem as relações cliente-terapeuta (Envolvimento, Conexão Emocional) e dimensões que se reportam às relações dentro da família (Segurança, Partilha de Objetivos), tal como estão evidenciadas diferentes áreas do funcionamento, cognitivo-comportamentais (Envolvimento, Partilha de Objetivos) e afetivas (Segurança, Conexão Emocional); b) transteórico porque reflete aspetos da aliança que não são exclusivos de uma teoria particular de psicoterapia (e.g., psicanalítica, sistémica, cognitiva) ou de um modelo específico de terapia familiar (e.g., estrutural, estratégico, centrado nas soluções), sublinhando assim o caráter comum e transversal da aliança nas terapias.

\section{Estudos em Portugal}

\section{Como foi desenvolvido/adaptado e validado?}

O facto de o SOFTA-o ter resultado de uma profícua colaboração transatlântica, entre os EUA e Espanha, fizeram dele um instrumento particularmente interessante a explorar no nosso país. Portugal e Espanha são países vizinhos e as semelhanças entre os dois territórios tornaram atraente o objetivo de estudar o SOFTA-o na nossa prática clínica, investigação e formação/supervisão de terapeutas familiares, respondendo assim à necessidade premente de promover a pesquisa científica na área da terapia familiar sistémica.

Os trabalhos relativos ao desenvolvimento e aplicação da versão observacional portuguesa do SOFTA (Sotero, Portugal, Cunha, Vilaça, \& Relvas, 2010) inserem-se no âmbito de um conjunto alargado de estudos efetuados entre 2010 e 2011. Os estudos aqui apresentados foram pioneiros e tiveram como objetivo proporcionar a utilização desta grelha de avaliação observacional da aliança terapêutica (versão clientes e versão terapeuta), explorando as suas propriedades psicométricas no que diz respeito à fiabilidade (e.g., acordo interobservadores) e validade (e.g., convergente e preditiva) (Relvas et al., 2010). Atualmente encontram-se em curso estudos que permitam aprofundar as características deste mé- 
todo de medição da aliança, nomeadamente ao nível da validade interna (e.g., análise fatorial exploratória) e da validade convergente entre o SOFTA-o e o SOFTA-s, bem como promover a investigação científica na área das terapias conjuntas.

De forma a garantir a equivalência linguística e conceptual do instrumento, a versão original espanhola do SOFTA-o foi submetida a um processo de tradução-retroversão composto por quatro passos: (a) $1^{\mathrm{a}}$ versão - resultou da tradução para a língua portuguesa, por parte de uma psicóloga com experiência clínica e formação na área sistémica; (b) $2^{a}$ versão - originada a partir da comparação entre a $1^{\text {a }}$ versão traduzida e a versão original espanhola efetuada por uma portuguesa bilingue especialista de saúde mental com formação sistémica; (c) retroversão da $2^{\mathrm{a}}$ versão (usando o método inverso) feita por um terceiro especialista ${ }^{1}$ bilingue na área sistémica; (d) $3^{\text {a }}$ versão - emergiu da verificação da equivalência do conteúdo da retroversão com o instrumento original concretizada pelo autor espanhol (Valentín Escudero), o qual apenas sugeriu alterações menores.

Por fim, as diversas versões foram avaliadas e comparadas por cinco profissionais de saúde mental com experiência clínica, em termos da equivalência semântica (gramática e vocabulário), conceptual (contexto cultural) e idiomática (coloquialismos) do conteúdo dos itens. Como resultado desta validação consensual foram feitas ligeiras alterações de linguagem e reformuladas algumas expressões para melhorar a compreensão dos itens, resultando deste processo a versão final do instrumento. Posteriormente foi conduzido um pré-teste de análise e interpretação dos descritores, através da leitura da grelha observacional, com uma amostra de quatro mestrandos de Psicologia Clínica, o qual não revelou dificuldades na compreensão dos enunciados. Esta análise prévia da versão final do instrumento teve como objetivo averiguar alguns aspetos da validade facial.

Paralelamente à tradução do instrumento, foi também realizada a tradução do Manual de Treino do SOFTA-o (Friedlander et al., 2005; versão traduzida e adaptada por Sotero et al., 2010), o qual contém a operacionalização detalhada dos descritores comportamentais, fornecendo

\footnotetext{
1 Agradecemos ao Prof. Jorge De Vega a sua colaboração neste trabalho.
} 
orientações específicas sobre o significado exato dos itens e os contextos em que devem ser registados. Este trabalho de tradução foi desenvolvido pela equipa de investigação, e posteriormente analisado e validado por um dos autores do instrumento.

\section{Estudos de precisão}

A fiabilidade traduz a precisão do método de medição e pode ser averiguada através da consistência ou estabilidade desse método (e.g., teste, instrumento, grelha de observação). $O$ atributo da consistência de uma medida raramente é uma questão de tudo ou nada (Cohen, Swerdlik, \& Smith, 1992), existindo graus de confiabilidade e várias formas de o medir (e.g., método teste-reteste, comparações split-half). No caso particular das metodologias observacionais o estabelecimento da fiabilidade é habitualmente feito a partir do cálculo da concordância entre dois ou mais avaliadores que observam o mesmo evento, registando-o de forma independente (Dessen \& Murta, 1997). Dito de outro modo, a fiabilidade interobservadores permite averiguar se diferentes observadores ao utilizarem os mesmos métodos de avaliação obtêm resultados consistentes, existindo vários índices de acordo interjuízes mencionados na literatura (e.g., coeficiente $S$ de Bennet, Alpert e Goldstein, o coeficiente Kappa de Cohen). Segundo Bakeman (2000), o acordo entre observadores pode ser visto como uma condição sine qua non da investigação observacional. $\mathrm{O}$ facto de se tratarem de dados observacionais recolhidos por observadores humanos, através de um juízo crítico, tornam especialmente importantes os aspetos relacionados com a formação e treino dos observadores, o acordo entre eles e a fiabilidade das dados recolhidos. A este propósito convém clarificar que acordo não é o mesmo que fiabilidade (Bakeman, 2000), dado que o acordo apenas mede a igualdade ou semelhança das observações entre avaliadores, enquanto a fiabilidade se reporta à possibilidade de generalização (ou reprodutibilidade) dos dados (Bakeman, 2000). No caso de investigações observacionais, um dos coeficientes de generalização, ou de fiabilidade, apropriados é o Coeficiente de Correlação 
Intraclasse (ICC do inglês Intraclass Correlation Coeficient) (Bakeman, 2000), o qual verifica a igualdade das classificações médias atribuídas por vários sujeitos (Pestana \& Gageiro, 2005).

Para incrementar e avaliar a fiabilidade da versão portuguesa do SOFTA-o foram conduzidos três estudos distintos, os quais se descrevem a seguir.

Estudo 1. De modo a procurar controlar e minimizar enviesamentos foram tomadas algumas medidas preventivas, nomeadamente uma fase de treino de codificação exaustiva e o uso do manual detalhado de codificação. Neste sentido, dois elementos da equipa de investigação receberam uma formação presencial no SOFTA-o, na Universidade da Corunha, durante dois dias, liderada pelo autor do instrumento (V. Escudero). Após a aquisição de uma boa compreensão do modelo do SOFTA e da discussão sobre as especificidades e dificuldades na utilização do instrumento, ambas as equipas de investigação (portuguesa e espanhola) observaram e cotaram em conjunto um vídeo de uma sessão de terapia familiar. Este processo envolveu repetidas comparações e acertos do processo de codificação entre as equipas, o que permitiu clarificar e aprofundar a descrição das unidades de codificação (i.e, dos descritores comportamentais). Apesar de não se ter constituído como um teste de fiabilidade formal, este procedimento proporcionou um aumento do acordo interjuízes no decorrer da aplicação do método de observação.

Estudo 2. A primeira prova formal de fiabilidade com a versão portuguesa do SOFTA-o realizou-se depois de um período de treino (entre 10-15 horas) com os vídeos disponíveis on-line no site do instrumento (Disponível em http://www.softa-soatif.com) e alguns vídeos de sessões de terapia familiar, representativos do material a analisar. Depois do treino conjunto, os elementos da equipa de investigação (dois doutorandos em Intervenção Familiar e dois mestres em Psicologia Clínica) observaram e codificaram, de forma independente, seis vídeos de sessões de terapia familiar. Dois elementos codificaram os comportamentos observáveis dos clientes (SOFTA-o, versão clientes) e outros dois codificaram os comportamentos observáveis dos terapeutas (SOFTA-o, versão terapeuta). 
A fiabilidade inter-juízes foi avaliada através da cálculo do ICC em cada uma das dimensões pontuadas. Os coeficientes de correlação encontrados são apresentados no Quadro 2 .

Quadro 2.

Coeficiente de correlação intraclasse (ICC): SOFTA-o

\begin{tabular}{|c|c|c|}
\hline Dimensões & Versão Clientes & Versão Terapeuta \\
\hline Envolvimento no Processo Terapêutico & .90 & .89 \\
\hline Conexão Emocional com o Terapeuta & .91 & .86 \\
\hline Segurança Dentro do Sistema Terapêutico & .93 & .95 \\
\hline Sentimento de Partilha de Objetivos na Família & .91 & .91 \\
\hline
\end{tabular}

Os resultados obtidos foram indicadores de uma boa fiabilidade interobservadores, tendo sido obtidos valores da correlação intraclasse próximos dos reportados pelos autores do SOFTA-o (desde .72 a .95; Friedlander, Escudero, Horvath et al., 2006).

Estudo 3. Com vista a avaliar a precisão do sistema de medição (i.e., o grau em que uma medida repetida sob condições inalteradas mostra os mesmos resultados - precision; Taylor, 1997), as equipas de investigação portuguesa e espanhola procederam à codificação, de modo independente, de uma sessão de terapia familiar (role-play didático) e compararam ambas as cotações. Esta prova de precisão, na qual o autor espanhol foi auditor, revelou uma percentagem de acordo de $70 \%$ no caso da cotação dos comportamentos dos clientes e de $90 \%$ na cotação dos terapeutas.

Estes estudos preliminares foram reveladores da utilidade da versão portuguesa do sistema de observação, atendendo aos níveis de precisão alcançados. De notar que, apesar de se ter encontrado alguma evidência de fiabilidade neste estudo, esta deve ser avaliada caso-a-caso, tal como é aconselhável que os membros da equipa de avaliadores reúnam periodicamente para compararem os seus resultados e não deixarem que o acordo e sincronismo na aplicação dos critérios de registo e de pontuação diminuam (Friedlander et al., 2005). 


\section{Estudos de validade}

A validade refere-se a uma propriedade do instrumento de medida - um instrumento é válido quando mede aquilo que pretende medir - enquanto a validação se mostra um processo complexo porque se trata de provar cientificamente a validade desse instrumento (Pasquali, 2007). A validade de constructo de um instrumento não se estabelece com a confirmação de um único estudo, pelo contrário requer um conjunto de resultados consistentes, a partir de um conjunto de estudos, implicando a acumulação de evidências científicas para verificar tal propriedade (Pasquali, 2007). Neste sentido, os estudos que a seguir se apresentam reportam-se apenas a alguns indicadores, sobretudo da validade de constructo, não dispensando futuras investigações complementares da validade do SOFTA-o. Nestes estudos de validade foram considerados os seguintes indicadores: (1) as intercorrelações entre as dimensões do SOFTA; (2) os dados da aliança nas terapias familiares com diferentes grupos de clientes (filhos adolescentes vs. pais); (3) a relação com os resultados terapêuticos (abandono vs. processos terminados).

Estudo 1. A associação entre as dimensões avaliadas pelo SOFTA foi determinada através do cálculo do grau de associação ou de intercorrelação entre as dimensões, utilizando o coeficiente de correlação de Spearman (medida de associação para variáveis ordinais; Maroco, 2007), no sentido de perceber o grau em que os indicadores designados para medir o mesmo constructo (i.e., aliança terapêutica) são relacionados e convergentes (convergent validity; Campbell \& Fiske, 1959). No caso dos indicadores terem uma relação forte entre si apresentarão correlações elevadas, acontecendo o inverso com os que não se relacionam. Para aceder às intercorrelações entre as quatro dimensões foi aleatoriamente selecionado um elemento de cada família de um conjunto de 40 famílias $(N=40)$, de modo a assegurar a independência dos dados. Conforme ilustrado no Quadro 3, as relações entre as quatro dimensões variam entre .05 e .38. A Conexão Emocional surge como a única dimensão que está significativamente associada a todas as outras, apesar de as correlações obtidas serem moderadas. Através do cálculo do coeficiente de determinação podemos 
concluir que a Conexão Emocional partilha 14\% da sua variância com o Envolvimento e igual percentagem de variância comum com a Segurança e com a Partilha de Objetivos, respetivamente. De notar que todas as dimensões se correlacionam positivamente entre si, conforme seria expectável atendendo ao constructo avaliado. As correlações mais baixas estão associadas à dimensão Segurança (Envolvimento $r_{s}=.05$ e Partilha de Objetivos $\left.r_{s}=.08\right)$. Este resultado vai ao encontro do que foi encontrado no estudo original (Friedlander, Escudero, Horvath et al., 2006), no qual a Segurança e a Partilha de Objetivos aparecem como as dimensões menos correlacionadas $\left(r_{s}=.18\right)$. Os resultados obtidos devem contudo ser interpretados com precaução, atendendo à reduzida dimensão da amostra $(N=40)$.

Quadro 3.

Intercorrelações entre as dimensões do SOFTA-o

\begin{tabular}{|c|c|c|c|c|}
\hline & ENV & $\mathrm{CE}$ & $\mathrm{S}$ & SPO \\
\hline Envolvimento no Processo & - & & & \\
\hline Conexão Emocional & $.38^{*}$ & - & & \\
\hline Segurança Dentro Sistema Terapêutico & .05 & $.37^{*}$ & - & \\
\hline Sentimento de Partilha de Objetivos & .26 & $.38^{*}$ & .08 & - \\
\hline
\end{tabular}

$\overline{\text { ENV }}=$ Envolvimento no Processo; $\mathrm{CE}=$ Conexão Emocional; $\mathrm{S}=$ Segurança Dentro Sistema Terapêutico; SPO = Sentimento de Partilha de Objetivos.

$* p<.05$

Estudo 2. Para determinar até que ponto os comportamentos observáveis, dentro de cada dimensão do SOFTA-o, são clinicamente significativos e discriminantes entre as alianças estabelecidas com pais e filhos adolescentes, respetivamente, foi conduzido um teste de validade com estes dois grupos de clientes no contexto da terapia familiar (validade grupos-conhecidos). Neste âmbito, 49 sujeitos (21 pais/mães e 28 filhos/as adolescentes) que participaram numa $1^{\text {a }}$ sessão de terapia familiar foram avaliados com o SOFTA-o, procedendo-se depois à comparação da força da aliança entre os grupos. Depois de comparados os resultados obtidos apontam para diferenças estatisticamente significativas na força da aliança nas dimensões Envolvimento no Processo Terapêutico $(z=-2.97, p=.003)$ e Conexão Emocional com o Terapeuta $(z=-2.98, p=.003)$. Nesta amostra, os adolescentes $(\mu=20.05)$ estavam menos envolvidos na terapia familiar do que os seus pais $(\mu=31.60)$, 
bem como se apresentavam menos conectados emocionalmente com o terapeuta ( $\mu_{\text {adolescentes }}=20.30 ; \mu_{\text {pais }}=31.26$ ) (Sotero, Relvas, \& Escudero, 2011). Estes resultados parecem corroborar os estudos que apontam para o facto de ser particularmente desafiante estabelecer uma relação terapêutica com adolescentes, uma vez que estes se apresentam com frequência no início da terapia familiar contra a sua vontade (Friedlander et al., 2006).

Estudo 3. A relação entre a aliança terapêutica e os resultados terapêuticos está teórica e empiricamente descrita na literatura, principalmente na terapia individual (Horvath et al., 2011), mas também na terapia familiar (Friedlander, Escudero, Heatherington, \& Diamond, 2011). Assim, foi conduzida uma prova de validade com o propósito de avaliar a capacidade do SOFTA-o prever algo que teoricamente deveria ser capaz de prever (predictive validity; Cronbach \& Meehl, 1955). Os resultados do estudo qualitativo com quatro casos clínicos contrastantes, do ponto de vista dos resultados da terapia familiar (conclusão da terapia por mútuo acordo vs. abandono da terapia) (Sotero et al., 2010), corroboraram a associação entre a aliança e os resultados terapêuticos encontrada em pesquisas anteriores (Beck et al., 2006). As famílias que terminaram a terapia por mútuo acordo apresentavam no início do processo alianças moderadamente fortes e congruentes nas quatro dimensões do SOFTA. Por oposição, nos casos em que houve abandono do processo foram obtidas avaliações notavelmente menos favoráveis nas quatro dimensões da aliança (Sotero, Portugal, Cunha, Vilaça, Alarcão et al, 2010).

\section{Aplicação}

\section{Como aplicar, cotar e interpretar?}

Em primeiro lugar, independentemente do objetivo de aplicação do instrumento (clínico ou investigação) é fundamental uma boa compreensão do modelo conceptual que está subjacente ao SOFTA. É também essencial que os codificadores ou observadores se familiarizem com as definições operacionais das dimensões e com os descritores comportamentais que lhes estão associados, apesar de não ser necessária a sua memorização. 
Embora não seja requisito ter experiência clínica, o SOFTA-o deve ser utilizado por observadores que sejam licenciados numa área da saúde mental e pós-graduados em terapia familiar ou intervenção sistémica. Quando os codificadores apresentarem um bom conhecimento acerca dos itens comportamentais e das dimensões deve seguir-se um período de treino de cotação. Os autores do instrumento sugerem a utilização dos vídeos de treino disponíveis em http://www.softa-soatif.com, os quais representam trechos de sessões terapêuticas ilustrativas de descritores comportamentais, positivos e negativos, em cada uma das dimensões da aliança. No caso da investigação, recomenda-se que, pelo menos, dois observadores avaliem a sessão de terapia familiar e que o treino de codificação com o SOFTA-o inclua a cotação prévia de 6 a 12 vídeos representativos do material a analisar, alcançando-se normalmente uma boa fiabilidade interobservadores depois de 10 a 15 horas de treino, durante 4 a 5 semanas.

Para além do material a analisar (sessão de terapia familiar/casal) é necessário que o codificador disponha do instrumento (SOFTA-o, versão clientes e/ou terapeutas) e do Manual de Treino (Friedlander et al., 2005; versão traduzida e adaptada por Sotero et al., 2010), do qual constam as instruções de codificação indispensáveis à aplicação das grelhas de observação e as definições operacionais de cada descritor comportamental. As definições dos descritores do terapeuta estão igualmente incluídas no manual e proporcionam aos observadores orientações específicas sobre quando e em que contexto devem ser registados.

A tarefa de avaliar a aliança terapêutica com o SOFTA-o comporta duas fases distintas: 1) registar a presença de indicadores comportamentais ao longo da sessão; 2) atribuir uma pontuação sobre a força da aliança para cada dimensão, imediatamente depois de ver a sessão completa. O pressuposto subjacente a este processo é que a pontuação atribuída a cada uma das quatro dimensões da aliança seja feita com base no registo de indicadores comportamentais observáveis que refletem sentimentos, pensamentos e atitudes internas dos membros da família sobre a terapia e o relacionamento com o terapeuta.

$\mathrm{Na}$ primeira fase os avaliadores observam de forma independente o vídeo, parando e rebobinando as vezes que forem necessárias. Quando se 
observa algum comportamento incluído na lista de descritores, como por exemplo "o cliente expressa otimismo ou indica que houve uma mudança positiva", o avaliador coloca uma marca no espaço em branco reservado para esse descritor e para esse membro da família (por exemplo, "mãe" ou "criança"). De forma a recordar, no final da sessão, a importância ou clareza e intensidade dos comportamentos assinalados podem ser colocadas notas ou comentários adicionais.

Na segunda fase, isto é, no final da sessão que está a ser avaliada, o observador deverá atribuir uma pontuação a cada membro da família presente na sessão, em cada uma das 3 dimensões (Envolvimento, Conexão Emocional, Segurança), dado que na dimensão Sentimento de Partilha de Objetivos na Família faz-se uma avaliação da família como um todo e, portanto, é dada uma pontuação para toda a família/casal.

As indicações que orientam a escala de pontuação referem-se à valência (positiva ou negativa), intensidade e frequência do comportamento, bem como à sua importância e significado no contexto da sessão. Cada dimensão é pontuada segundo uma escala numérica/ordinal de sete pontos $(-3,-2,-1,0,+1,+2,+3)$ : $O$ (i.e., nada a assinalar ou aliança neutra), -3 representa o extremo negativo (i.e., aliança muito problemática) e +3 o outro extremo (i.e., aliança muito forte). A pontuação de 0 é dada quando não há comportamentos positivos ou negativos registados ou quando o número e a natureza desses comportamentos se anulam uns aos outros. Assim, uma pontuação de 0 sugere que a aliança não é particularmente positiva ou particularmente negativa. Quando apenas existem comportamentos positivos a pontuação deve ser $+1,+2$ ou +3 . De forma similar, quando apenas há comportamentos negativos, a pontuação deve ser -1, -2 ou -3. A subjetividade existe quando é necessário considerar o contexto, o conteúdo e o significado dos comportamentos observados, e a pontuação requer uma maior inferência quando os clientes (ou terapeutas) apresentam durante a sessão comportamentos quer positivos quer negativos. Nestas circunstâncias, a pontuação deve ser entre $-1,0 \mathrm{e}+1$.

A pontuação atribuída a cada membro da família pode ser influenciada pela forma como são pontuados os outros membros da família. Por essa razão, o avaliador deve em primeiro lugar atender às marcas feitas para 
assinalar os descritores comportamentais e decidir quem é o membro da família menos envolvido na terapia. Esse membro deve ser avaliado e pontuado primeiro, passando-se depois à segunda pessoa menos envolvida e assim sucessivamente. Desta maneira, o membro da família mais envolvido ou comprometido com a terapia é avaliado em último lugar.

Com efeito, a ponderação final não é feita pelos avaliadores confiando simplesmente na sua intuição ou impressão final para pontuar cada dimensão, mas tem que se basear e apoiar nos descritores comportamentais identificados e assinalados durante a sessão, atendendo também ao conteúdo e ao contexto. Por exemplo, o indicador "os membros da família oferecem um acordo para um compromisso" terá um significado diferente se o compromisso estiver diretamente relacionado com o problema que está em discussão ou caso se refira a algo periférico relativamente às preocupações centrais da família. Consideremos um outro exemplo, reportando-nos a um dos vídeos de treino acessíveis on-line. Um jovem rapaz está sentado quase na posição fetal, escondendo a cabeça e a parte superior do corpo. Parece relutante em responder ao terapeuta ou à avó, que fala para ele de forma suave a carinhosa. A determinada altura, o rapaz revela algo doloroso: "O meu pai bate-me". Poucos minutos depois, começa a chorar. Nestes dois minutos de role-play, os comportamentos negativos de Segurança são (a) o rapaz apresenta uma postura corporal defensiva e (b) está relutante em responder à avó quando esta lhe fala diretamente. Um indicador positivo de Segurança é a sua expressão de vulnerabilidade (i.e., revela algo doloroso e chora). Dado que os comportamentos extremos negativos superam o positivo, a pontuação adequada era -1. Se o rapaz não tivesse chorado ou mencionado o abuso físico (i.e., não tivesse manifestado um comportamento positivo de Segurança), a pontuação deveria ser superior a -1 e baseada apenas nos comportamentos não-verbais. Contudo, se ele estivesse relutante em responder mas não estivesse sentado de forma tão defensiva, a pontuação dada poderia ser +1 , dada a natureza sensível da sua revelação. Se ele tivesse chorado, mencionado que o pai lhe batia, sem estar numa postura defensiva nem relutante (i.e. se apenas apresentasse indicadores positivos de Segurança), a pontuação deveria ser +3 . 


\section{Vantagens, limitações e estudos futuros}

É hoje aceite que a aliança terapêutica é uma variável crítica no processo e nos resultados da terapia familiar e de casal (Friedlander et al., 2011). Todavia, a sua construção e manutenção no contexto das terapias conjuntas é um processo complexo, multipessoal e multigeracional, sendo ainda diminuto o conhecimento científico a seu respeito. Assim, uma primeira vantagem do SOFTA-o é disponibilizar em português uma ferramenta que permite "ver" a(s) aliança(s) terapêutica(s) com base num modelo conceptual derivado da teoria, investigação e prática clínica. Outra vantagem, é o facto de o SOFTA-o possibilitar a identificação e codificação dos comportamentos observáveis dos clientes enfatizando o processo de interação social (cliente-terapeuta; cliente-cliente; cliente-família), momento-a-momento, na construção da(s) aliança(s) terapêutica(s). Para além disso, este instrumento disponibiliza ainda uma versão que permite identificar os comportamentos dos terapeutas que contribuem (mais e menos) para a construção e manutenção da aliança ao longo das sessões. A representação da experiência clínica, nomeadamente dos comportamentos dos terapeutas que contribuem para a aliança e dos comportamentos dos clientes que a revelam, constitui-se assim como um método bastante útil e sistemático que pode ser utilizado na investigação, na formação e treino de terapeutas, bem como na prática clínica.

Apesar das vantagens referidas, há, contudo, algumas limitações neste sistema de observação que devem ser tomadas em consideração. Primeiro, embora se aceite que os pensamentos e sentimentos acerca da aliança se manifestam em determinados comportamentos expressos, não pode ser assumido que os comportamentos representados no SOFTA-o representam o universo dos comportamentos-aliança dos clientes ou dos comportamentos-contributo dos terapeutas. Para além disso, alguns dos comportamentos predeterminados, tais como "O cliente expressa fisicamente afeto ou carinho pelo terapeuta" (Conexão Emocional) e "O cliente menciona de forma ansiosa/incómoda a câmara, os observadores, a supervisão, ou os procedimentos de investigação" (Segurança), não ocorrem em todos os contextos clínicos ou com os clientes de todas 
as culturas (Friedlander et al., 2006). Desta forma, os descritores comportamentais contemplados em cada uma das dimensões do SOFTA refletem a maioria das abordagens terapêuticas na terapia familiar e de casal, mas inevitavelmente limitam o que é visto e registado. Uma segunda assunção que deve ser feita reporta-se ao facto de as alianças terapêuticas serem bidirecionais. De acordo com o SOFTA-o há comportamentos que identificam uma aliança forte e outros que assinalam uma aliança fraca, sendo também possível avaliar uma aliança como neutra, quando não existem indicadores comportamentais suficientemente positivos ou negativos, ou quando ambos se anulam. Dada a natureza ordinal da escala, não é possível considerar, por exemplo, que quando um cliente obteve no Envolvimento uma pontuação de +2 (aliança bastante forte), significa que está duas vezes mais envolvido na terapia do que um outro que recebeu a pontuação de +1 (aliança ligeira). Finalmente, não podemos assumir que todos os indicadores comportamentais do instrumento são igualmente indicativos da força da aliança. Ter uma postura corporal “aberta”, por exemplo, não é tão significativo como revelar um segredo familiar, no caso da dimensão Segurança. Assim, registar simplesmente a frequência dos indicadores comportamentais não é suficiente, sendo necessário um julgamento crítico que tome em conta o conteúdo (verbal ou não verbal) e os contextos interpessoais. Aspetos como o tom e o volume da voz do cliente, o seu comportamento não-verbal, ou as reações dos outros elementos da família, fornecem pistas acerca da força e importância dos descritores comportamentais no decorrer do contexto terapêutico. É importante não esquecer que a recolha observacional dos dados é feita por observadores treinados e implica a realização de julgamentos e inferências, baseados na observação a partir das grelhas de registo ("buman synthesizer", Friedlander et al., 2006, p. 264), algo não muito diferente daquilo que o terapeuta faz ao longo da terapia mas de forma menos sistemática.

Em termos de investigação futura será importante prosseguir com os estudos de evidência de validade no contexto português, nomeadamente testar o modelo resultante de uma análise fatorial confirmatória e realizar estudos de validade convergente com outros instrumentos de medida da 
aliança terapêutica (e.g., SOFTA-s; VTAS-R). A investigação com o SOFTA-o permitirá ainda, futuramente, desenvolver uma visão crítica sobre os descritores comportamentais que compõe cada uma das dimensões da aliança e sobre a sua adequação ao contexto da terapia familiar em Portugal.

\section{Bibliografia}

Bakeman, R. (2000). Behavioral observation and coding. In H. T. Reis \& C. K. Judd (Eds.), Handbook of research methods in social psychology (pp. 138-159). New York: Cambridge University Press.

Beck, M., Friedlander, M., \& Escudero, V. (2006). Three perspectives of clients' experiences of the therapeutic alliance: A discovery-oriented investigation. Journal of Marital and Family Therapy, 32, 355-368. doi: 10.1111/j.1752-0606.2006.tb01612.x

Bertalanffy, L. Von (1972). Théorie générale des systèmes. Paris: Dunod. Edição original 1986.

Bordin, E. S. (1979). The generalizability of the psychoanalytic concept of the working alliance. Psychotherapy, 16, 252-260. doi: 10.1037/h0085885

Campbell, D. T., \& Fiske, D. W. (1959). Convergent and discriminant validation by the multitrait multimethod matrix. Psychological Bulletin, 56, 81-105. doi: 10.1037/h004616

Christensen, L. L., Russell, C. S., Miller, R. B., \& Peterson, C. M. (1998). The process of change in couples therapy: A qualitative investigation. Journal of Marital and Family Therapy, 24, 177-188. doi: 10.1111/j.1752-0606.1998.tb01074.x

Cohen, R. J., Swerdlik, M. E., \& Smith, D. K. (1992). Psychological testing and assessment: An introduction to tests and measurement ( $2^{\text {nd }}$ ed.). California: Mayfield Publishing Company.

Cronbach, L. J., \& Meehl, P. E. (1955). Construct validity in psychological tests. Psychological Bulletin, 52, 281-302. doi: 10.1037/h0040957

Dessen, M. A., \& Murta, S. G. (1997). A metodologia observacional na pesquisa em psicologia: Uma visão crítica. Cadernos de Psicologia, 1, 47-60.

Diamond, G. M., Liddle, H. A., Hogue, A., \& Dakof, G. A. (1999). Alliance-building interventions with adolescents in family therapy. A process study. Psychotherapy, 36, 355-368. doi: $10.1037 / \mathrm{h} 0087729$

Escudero, V. (2009). La creación de la alianza terapéutica en la terapia familiar. Apuntes de Psicologia, 27, 247-259.

Escudero, V., Friedlander, M. L., Varela, N., \& Abascal, A. (2008). Observing the therapeutic alliance in family therapy: Associations with participants' perceptions and therapeutic outcomes. Journal of Family Therapy, 30, 194-214. doi: 10.1111/j.1467-6427.2008.00425.x

Escudero, V., Heatherington L., \& Friedlander, M. L. (2010). Therapeutic alliances and alliance building in family therapy. In J. C. Muran \& J. P. Barber (Eds.), The therapeutic alliance. An evidenced-based guide to practice (pp. 240-262). New York: Guilford.

Fallon, I. (1991). Behavioral family therapy. In A. Gurman \& D. Kniskern (Eds.). Handbook of family therapy (Vol. II, pp. 75-85). New York: Brunner/Mazel.

Friedlander, M. L., Bernardi, S., \& Lee, H. (2010). Better versus worse family therapy sessions as reflected in clients' alliance-related behavior. Journal of Counseling Psychology, 57, 198-204. doi: 10.1037/a0019088 
Friedlander, M. L., Escudero, V., \& Heatherington, L. (2006). Therapeutic alliances in couple and family therapy: An empirically informed guide to practice. Washington, DC: American Psychological Association.

Friedlander, M., Escudero, V., Heatherington, L., Deihl, L., Field, N., Lehman, P., . . Cutting, M. (2005). Sistema de Observación de la Alianza Terapéutica en Intervención Familiar (SOATIF_o) - Manual de Entrenamiento - revisado. Acedido em 12, janeiro, 2009, em http://www.softa-soatif.com/docusofta/softa\%20instruments/manuales/SOATIFManual. pdf. Tradução Portuguesa de Sotero L., Portugal A., Cunha D., Vilaça M. \& Relvas, A. P. (2010). Universidade de Coimbra: Material não publicado.

Friedlander, M. L., Escudero, V., Heatherington, L., \& Diamond, G. M. (2011). Alliance in couple and family therapy. Psychotherapy, 48, 25-33. doi: 10.1037/a0022060

Friedlander, M. L., Escudero, V., Horvath, S., Heatherington, L., Cabero, A., \& Martens, M. P. (2006). System for Observing Family Therapy Alliances: A tool for research and practice. Journal of Counseling Psychology, 53, 214-225. doi: 10.1037/0022-0167.53. 2.214

Goldfried, M. R. (1980). Toward the delineation of therapeutic change principles. Applied and Preventive Psychology, 13, 3-4.

Heatherington, L., \& Friedlander, M. L. (1990). Couple and family therapy alliance scales: Empirical considerations. Journal of Marital and Family Therapy, 16, 299-306. doi: 10.1111/j.1752-0606.1990.tb00851.x

Horvath, A. O., \& Bedi, R. P. (2002). The alliance. In J. C. Norcross (Ed.), Psychotherapy relationships that work: Therapist contributions and responsiveness to patients (pp. 37-69). New York: Oxford University Press.

Horvath, A. O., Del Re, A., Flückiger, C., \& Symonds, D. B. (2011). Alliance in individual psychotherapy. Psychotherapy, 48, 9-16. doi:10.1037/a0022186

Horvath, A. O., \& Greenberg, L. S. (1986). The development of the Working Alliance Inventory. In L. S. Greenberg \& W. M. Pinsof (Eds.), The psychotherapeutic process: A research handbook (pp. 529-556). New York: Guildford.

Horvath, A. O., \& Symonds, B. D. (1991). Relation between working alliance and outcome in psychotherapy: A meta-analysis. Journal of Counseling Psychology, 38, 139-149. doi: 10.1037/0022-0167.38.2.139

Knobloch-Fedders, L. M., Pinsof, W. M., \& Mann, B. J. (2004). The formation of the therapeutic alliance in couple therapy. Family Process, 43, 425-442. doi: 10.1111/j.1545$-5300.2004 .00032 . x$

Luborsky, L., Crits-Cristoph, P., Alexander, L., Margolis, M., \& Cohen, M. (1983). Two helping alliance methods for predicting outcomes of psychotherapy: A counting signs vs. global rating method. Journal of Nervous and Mental Disease, 171, 480-491.

Luborsky, L., Singer, B., \& Luborsky, L. (1975). Comparative studies of psychotherapies; "Is it true that everybody has won and all must have prizes"? Archives of General Psychiatry, $32,995-1008$.

Maroco, J. (2007). Análise estatística com utilização do SPSS. Lisboa: Sílabo.

Martin, D. J., Garske, J. P., \& Davis, K. M. (2000). Relation of the therapeutic alliance with outcome and other variables: A meta analytic review. Journal of Clinical and Consulting Psychology, 68, 438-450. doi: 10.1037/0022-006X.68.3.438

Morin, E. (1991). Introdução ao pensamento complexo (D. Matos, Trad.). Lisboa: Instituto Piaget. (Obra Original Publicada em 1990).

Muñiz de la Peña, C., Friedlander, M. L., \& Escudero, V. (2009). Frequency, severity, and evolution of split family alliances: How observable are they? Psychotherapy Research, 19, 133-142. doi: 10.1080/10503300802460050 
Pasquali, L. (2007). Validade dos testes psicológicos: Será possível reencontrar o caminho? Psicologia: Teoria e Pesquisa, 23, 99-107.

Pestana, M. H., \& Gageiro, J. N. (2005). Análise de dados para ciências sociais - A complementaridade do SPSS ( $4^{\mathrm{a}}$ ed.). Lisboa: Sílabo.

Pinsof, W. B. (1994). An integrative systems perspective on the therapeutic alliance: Theoretical, clinical, and research implications. In A. O. Horvath \& L. S. Greenberg (Eds.), The working alliance: Theory, research, and practice (pp. 173-195). New York: Willey.

Pinsof, W. B. (1995). Integrative problem-centered therapy. New York: Basic Books.

Pinsof, W. B., \& Catherall, D. (1986). The integrative psychotherapy alliance: Family, couple, and individual therapy scales. Journal of Marital and Family Therapy, 12, 137-151. doi: 10.1111/j.1752-0606.1986.tb01631.x

Pinsof, W. M., Zinbarg, R., \& Knobloch-Fedders, L. M. (2008). Factorial and construct validity of revised short form Integrative Psychotherapy Alliance Scales for family, couple and individual therapy. Family Process, 47, 281-301. doi: 10.1111/j.1545-5300.2008.00254.x

Rait, D. S. (2000). The therapeutic alliance in couples and family therapy. Journal of Clinical Psychology, 56, 211-224. doi: 10.1002/(SICI)1097-4679(200002)56:2<211::AID$-\mathrm{JCLP} 7>3.0 . \mathrm{CO} ; 2-\mathrm{H}$

Relvas, A. P., Escudero, V., Sotero, L., Cunha, D., Portugal, A., \& Vilaça, M. (2010). The System for Observing Family Therapy Alliances (SOFTA) and the preliminary Portuguese studies. $8^{\text {th }}$ Electronic EFTA Newsletter. Retrieved from http://www.eftacim.org/doc_pdf/softa.pdf

Robbins, M. S., Liddle, H. A., Turner, C. W., Dakof, G. A., Alexander, J. F., \& Kogan, S. M. (2006). Adolescent and parent therapeutic alliances as predictors of dropout in multidimensional therapy. Journal of Family Psychology, 20, 108-116. doi: 10.1037/0893$-3200.20 .1 .108$

Robbins, M. S., Turner, C. W., Alexander, J. F., \& Perez, G. A. (2003). Alliance and dropout in family therapy for adolescents with behavior problems: Individual and systemic effects. Journal of Family Psychology, 17, 534-544. doi: 10.1037/0893-3200.17.4.534

Rogers, C. R., \& Wood, J. K. (1974). Client-centered theory: Carl R. Rogers. In A. Burton (Ed.), Operational theories of personality (pp. 211-258). New York: Brunner/Mazel.

Sotero, L., Portugal, A., Cunha, D., Vilaça, M., Alarcão, M., Escudero, V., \& Relvas, A. P. (2010, October). Exploratory Study of Preliminary Portuguese Data with SOFTA-o in Systemic Family Therapy. Oral Communication presented at the $7^{\text {th }}$ European Family Therapy Association (EFTA) Congress, Paris, France. Retrieved from http://www. europeanfamilytherapy.eu/wp-content/uploads/2012/10/sotero.pdf

Sotero, L., Portugal, A., Cunha, D., Vilaça, M., \& Relvas, A. P. (2010). Sistema de Observação da Aliança em Terapia Familiar: SOFTA [Versão Portuguesa. Versão Clientes; Versão Terapeutas]. Instrumento não publicado.

Sotero, L. \& Relvas, A. P. (2012). A intervenção com clientes involuntários: complexidade e dilemas. Psicologia \& Sociedade, 24, 187-196. doi:10.1590/S0102-71822012000100021

Sotero, L., Relvas, A. P., \& Escudero, V. (2011, July). The Therapeutic Alliance With Involuntary Adolescent Clients in the Context of Systemic Family Therapy. In Gary Diamond (Chair), Adolescents in Family Therapy: The Challenge of Building Therapeutic Alliances. Symposium conducted at the International Meeting of the $42^{\text {nd }}$ Society for Psychotherapy Research (SPR), Bern, Switzerland.

Symonds, B. D., \& Horvath, A. O. (2004). Optimizing the alliance in couple therapy. Family Process, 43, 443-455. doi: 10.1111/j.1545-5300.2004.00033.x

Taylor, J. R. (1997). An introduction to error analysis: The study of uncertainties in physical measurements ( $2^{\text {nd }}$ ed.). Sausalito: University Science Books. 